International Journal of Engineering \& Technology, $5(x)(2017) x x x-x x x$
International Journal of Engineering \& Technology
SPC
Website: $w w w . s c i e n c e p u b c o . c o m / i n d e x . p h p / I J E T$
doi:
Research paper, Short communication, Review, Technical paper

\title{
Conceptual Oriented Study on the Cloud Computing Architecture for the Full-Security
}

\author{
Dr.K.SAI MANOJ ${ }^{1 *}$, Ms.K.Mrudula ${ }^{2}$,G.Maanasa ${ }^{3}$, K.Phani Srinivas ${ }^{3}$ \\ ${ }^{1} \mathrm{CEO}$, Innogeecks Technologies and Amrita Sai Institute of Science and Technology, Vijayawada, AP, India \\ ${ }^{2}$ Managing Director, Innogeecks Technologies, Vijayawada, AP, India \\ ${ }^{3}$ Research Scholar, Acharya Nagarjuna University, Guntur Dist, AP, India \\ ${ }^{4}$ Associate Professor and Head R\&D, Amrita Sai Institute of Science and Technology, Vijayawada, AP, India
}

*E-mail: sai.kudaravalli@gmail.com

\begin{abstract}
Many developers have designed their own architecture for installing the cloud computing infrastructure. The existing cloud computing architectures differs in many ways. Security has been the serious issue as client related data and processing is undertaken using the infrastructure provided by third party service providers varies greatly. It is necessary to know the extent of inclusion of security into the cloud computing system and then find the best architecture that includes best and tight security system.

In this paper, a comparison of existing architectures from the perspective of inclusion of security infrastructure within cloud computing system is presented along with a comprehensive architecture that is included with every aspect of security taking into account the most of the vulnerabilities
\end{abstract}

Keywords: Cloud computing, security infrastructure, cloud computing architectures, enforcing security among cloud computing systems.

\section{Introduction}

Cloud computing is basically service model deployed over internet to provided services to the end users. The main objective of cloud computing technology is to ensure availability, high reliability and scalability of infrastructural facilities which include hardware, software, platforms, services and software which could be distributed to different computing locations. No standard definition as such has been defined and being followed as yet.

\section{Related work}

Various kinds of components are considered into a cloud computing system architecture which essentially explains the way the components are structured and the way the communication happens among the components. The components considered and placed in the architecture include cloud resources, services, middleware, application and system software. Nothing about the hardware as such is described in architecture. The architecture explains the properties of the software objects and the relationships between the objects.

The architecture that explains the cloud computing infrastructure shall deal with various aspects that include Xaas Structures, platform adaption, structuring cloud services, structuring cloud components, relationship between various components, Communicating between the objects, middleware that address the heterogeneity that exists among the communicating objects or to work like a broker between the user and the backend computing system, Deploying and managing security components and to be able check vari- ous legal issue especially when related to exporting data across the continents[1].

\section{Literature Survey:}

Many components exist within the cloud infrastructure. Data storage and retrieval is made available as a service the user. When it comes to the data confidentially of the same is important. Every component has an architecture that is built into overall cloud computing architecture. Many storage devices that are either supported on the servers or the network storage devices are clustered to form the total storage. Dedicated software running on one of the server is made responsible to manage the storage in terms of allocation, storing the user data and retrieval of the data as per user requirements. The software also deals with providing access rights to the user for availing the data services and revoking the same when the service is completed.

Security has been the most important issue when it comes to data storage and retrieval and also transmitting the same to the end user over the internet. Security is also the issue when the Data transfer from the user to the cloud is implemented.

Cloud storage is not just the data supported on a server as in a conventional system. Cloud storage as such includes network devices, storage devices, servers, applications, public access interface, client programs and similar such systems. A sub-system is implemented that manages the storage in the cloud computing environment

Clustered storage architecture is generally implemented for providing efficient storage services and also to support scalability and fault tolerant data storage and retrieval systems. The cloud storage resources are generally organized as a cluster, a grid or a distribut- 
ed file system. The connecting network and the storage management software together provide the data storage services to the end users.

Providing the user the easy access and high performance data services are the main objectives of cloud computing systems when it comes to the data storage and retrieval. The storage infrastructure provided in cloud computing systems is homogenous and supported on homogenous platforms while that not being the case now. A heterogeneous cloud storage infrastructure management has been presented [2]

Customers enters into service level agreements (SLA) with cloud computing service providers for want of various kind of service that are related infrastructure, platform and software provisioning. The services required and the quality of the services required differs a lot from user to user. Resource management systems generally do not follow SLA for managing the resources leading to some kind of rendering less quality services to the end users. SLA based resource allocation provides very high quality of fata storage services.

Customer driven approaches are mostly needed for providing quality services to the end users. The customer oriented service management, management of computational risk, autonomic resource management needs to be integrated into market base resource management. This will help in adapting to the rapidly changing services requirement scenario.

An architecture has been presents that considers the management of the requires in accordance with SLAs. Architecture has been proposed which is based on market driven policies and virtualization technologies which supports in providing flexible allocation of services [3].

Virtualized services which are dynamically scalable are provided to the users through a service model supported by cloud computing technologies. No standard as such exists as on date for implementing cloud computing solutions. Much architecture is being used for building cloud computing systems. The requirements of the users are to be found first and then classify the same based on some criteria. It has been shown that some features required by the users such as the requirements for storage, software, platform, securing the data etc., play a vital role in defining the architectures which should be used for building cloud computing infrastructure [4].

\section{Main Concept in this Paper:}

In this paper, we would like to consider important architectural requirements for Cloud computing systems from the point of view of enterprise users, such as infrastructure, storage, scalability, compliance and security. These considerations provide a guideline for creating architectural mechanism that will be helpful for software architects and developers while designing Cloud based applications.

\section{Investigations and Findings}

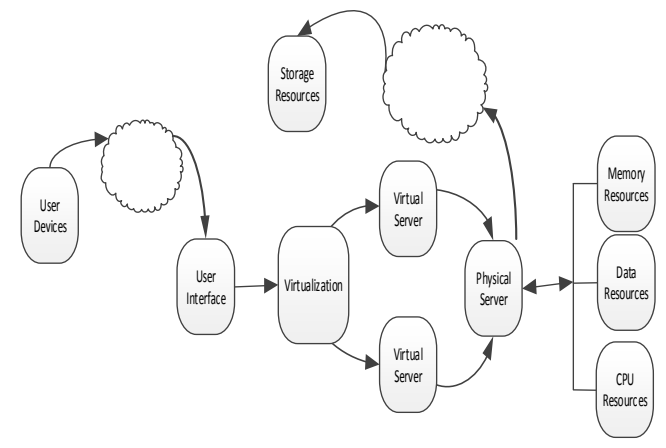

Figure 1

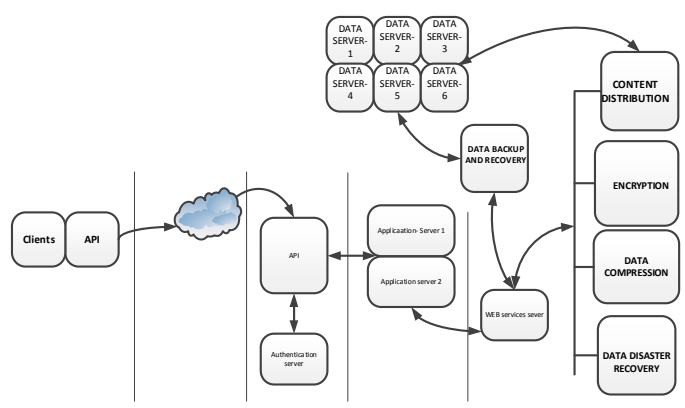

Figure 2

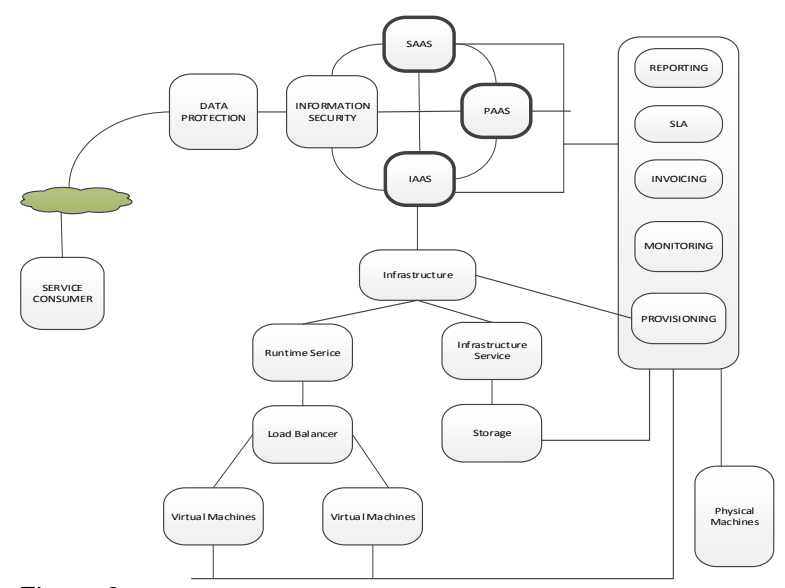

Figure 3

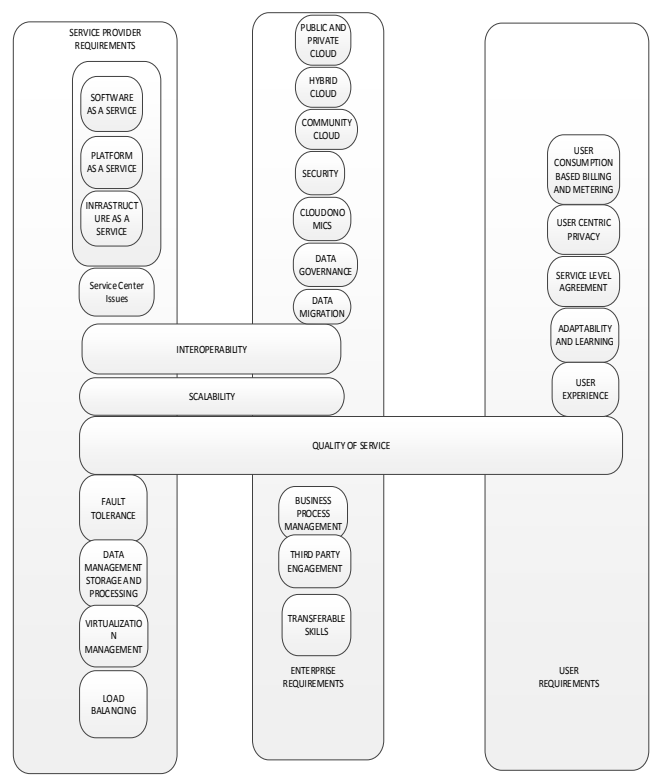

Figure 4

Conclusion:

Comparison of existing architectures from the perspective of inclusion of security infrastructure within cloud computing system was Investigated along with a comprehensive architecture that is included with every aspect of security taking into account the most of the vulnerabilities.

Author's Profiles:

Authors' contributions The other of the paper do all the work, the environment for research work are done by my best of my knowledge and supporting my family members.

Acknowledgements

This paper heartily dedicated to beloved Honble Secretary and Correspondent Sri. K.Ram Mohan Garu, \& Smt.K.Bhavani Devi Garu Amrita Sai Institute of science and technology. Also to all the respected 
Amrita Sai Management members. Our special thanks to the Innogeecks technologies, Vijayawada for their technical support in all the aspects. Also we would like to thank all the Industrial experts and eminent scientists for their constant technical support.

\section{REFERENCES}

1. Gerald Kaefer, Cloud Computing Architecture, Corporate Research and Technologies, Munich Germany, 4th Generation Data centre IEEE Spectrum,1-9,2010.

2. Dejun Wang, An Efficient Cloud Storage Model for Heterogeneous Cloud Infrastructures, JOURNAL, 1877 7058/10.1016, 510-515,2011.

3. Rajkumar Buyya1,2, Saurabh Kumar Garg1, and Rodrigo N. Calheiros, SLA-Oriented Resource Provisioning for Cloud Computing:Challenges, Architecture and Solutions,2011 International Conference on Cloud and Service Computing,1-10,2011.

4. Bhaskar Prasad Rimal · Admela Jukan -Dimitrios Katsaros . Yves Goeleven, Architectural Requirements for Cloud Computing, JOURNAL/10.1007/DOI 10.1007/s10723-010-9171-y,1-26,2011.

5. Kun Liua, Long-jiang Donga,"Research on Cloud Data Storage Technology and Its Architecture Implementation",JOURNAL/1877-7058/10.1016,133-137,2012.

6. Yuri Demchenko, Canh Ngo, Marc X. Makkes, Rudolf Strijkers, 1Cees de Laat, Defining Inter-Cloud Architecture for Interoperability and Integration, CLOUD COMPUTING 2012 : The Third International Conference on Cloud Computing, GRIDs, and Virtualization,ISBN: 978-161208-216-5,1-7,2012

7. Demeke Gebresenbet Bayyou member IEEE1, Dr. Menchita F. Dumlao, Cloud Computing Reference Architecture from Different Vendors Perspective, International Journal of Emerging Technology and Advanced Engineering,Vol:3/lssue:11,17,2013.

8. Rajkumar Buyya , Rodrigo N. Calheiros , Jungmin Son , Amir Vahid Dastjerdi, and Young Yoony, SoftwareDefined Cloud Computing: Architectural Elements and Open Challenges,3rd International Conference on Advances in Computing, Communications and Informatics (ICACCI) ,1-12,2014

9. A Survey on Protection of Multimedia Content in Cloud Computing Dr. K.Sai Manoj1, Mrudula Kudaravalli2, K Phani Srinivas3International Journal of Computer Science and Mobile ComputiVol.6 Issue.11, November- 2017, pg. 7-11

\section{Authors' information}

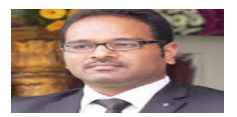

Dr. SAI MANOJ KUDARAVALLI, is a Founder and CEO in Innogeecks Technologies, Vijayawada and also acting as CEO for the Amrita Sai Institute of Science and Technology since 2014, and he played vital key role in Fidelity Investments as a Senior Business Analyst for 4.4 years in Business Analytics \& Research and worked as Project Engineer in Wipro Technologies for 1.5 years, He got more than 10 years of experiences in financial services, IT services and education domain.

He completed a Bachelor of Technology in Mechanical Engineering from Amrita University, Coimbatore. He is completing a Master of Technology in Information Technology from IIIT- Bangalore. He holds Doctor of Philosophy $(\mathrm{PhD})$ in the cloud computing arena from Kanpur University, India.

He filed three Provisional Patents. He was certified in Microsoft Certified Technology Specialist (MCTS) from Microsoft Corporation, and Certified Ethical Hacker v9 (CEH), and "Paul Harris Fellow" recognized by Rotary International. He has published more than 10 research papers in various reputed International and national research journals/conferences/ Magazines. He attended 4 national level workshops and participated 3 international workshops; He is also a charted Engineer (Computer Science) from IEI.

He is an active member of IEEE, ACM, IEI, SHRM, NEN - Bangalore Chapter, HR Sangham - Chennai, CCICI (Cloud Computing), Rotary International Services. He had certified as a Professional with various top organizations as Face book Certified Planning Professionalism Block chain foundation developer, GIAC Certified Web Application Defender (GWEB),AWS Certified Solutions Architect - Associate .Google Adwords Certification, Google Analytic Certification, Google Analytics Individual Qualification, Chartered Engineer ( C.Eng.).Microsoft Certified Technology Specialist (MCTS), CEH v9 (Certified Ethical Hacker v9)

Ms. K. MRUDULA working as a Managing Director in the Innogeecks Technologies. She was completed M. Tech from IIIT-Hyderabad. She got more than 6 years of experience in Teaching. She published more than 5 research papers in various International and national research journals. She attended 2 FDPs, and 1 workshop.

Mrs.G.Maanasa worked as HR manager in Jaya lakshmi Powercorp Ltd for a Period of 6 years after completing her M.B.A from RVR\&JC college of Engineering. She is currently Pursuing her doctorate (Ph.d) in Development of Framework for Tourism Promotion in AP and ICT Integration from Nagarjuna University.

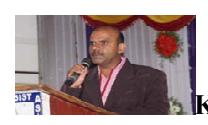
trial Experience as a team Leader in the research areas of Embedded Systems and Tele-Communications and also He is having about 18 years of Experience in Academics, Research and Industry. He received several research awards like Best Engineer Award, Best Teacher Award and Best Research Paper Award.

The Focus of His research work is Design of Patch antennas which are Suitable for Defense and Space Based Applications. He received an appreciation award in various National and International Conferences. He received Best Coordinator Certificates from IUCEE, IIT ROORKEE, IIT Bhubaneswar, NCAT, ELAT and INTEL. He attended

WIPRO Training Program. He completed one Joint research Program with

IIT Kharagpur. He organized various student level Competitions, workshops, Faculty Development Programs, Guest lectures, Orientation Programs, and Subject Based Seminars with scientists and Academicians. He is doing research work under the valuable Directions of Eminent Scientists He had done technical Discussions with experts at the Space Station, Antenna Research Lab, and Radar station. He Published research articles in Various Scopus International Journals. He is an active Editor/Reviewer for so many reputed international journals. He is acting as a resource Person/Visiting Professor for so many Industries, Scientific research labs and Engineering Institutions. He had selected for the most prestigious GLOBAL TEACHER CONGRESS AND AWARDS 2018 with the result Id: GTA18IN0834. He got appreciation from the eminent organizations for the role of technical session chair and also for the quality of the Creative Presentations 